\title{
Underlying Causes and Risk Factors of Chronic Renal Allograft Dysfunction Among Renal Transplant Recipients
}

\section{Göksel Güven', [MD] \\ ORCID: 0000-0002-6916-8907}

Şeref Rahmi Yılmaz', [MD]

ORCID: 0000-0002-5832-0336

Tolga Yıldırım', [MD]

ORCID: 0000-0001-7972-8317

Fazıl Tuncay Aki', [MD]

ORCID: 0000-0002-3625-9968

Yunus Erdem', [MD]

ORCID: 0000-0002-5389-3143
'Hacettepe University Faculty of Medicine, Department of Internal Medicine, Ankara, Turkey.

${ }^{2}$ Hacettepe University Faculty of Medicine, Department of Urology, Ankara, Turkey.

Corresponding Author: Göksel Güven Hacettepe University Faculty of Medicine, Department of Internal Medicine, Ankara, Turkey.

E-mail: drgoksel@hotmail.com

https://doi.org/10.32552/2021.ActaMedica.572

\section{w ABSTRACT Con}

Objective: Dialysis or renal transplantation are the two treatment options for end-stage renal disease patients. Renal transplantation from an appropriate donor increases survival and quality of life compared to treatment with dialysis. Recent advances in immunosuppressive therapy have significantly improved the success in 1-year graft survival. However, the long-term graft survival remains the same. Therefore, we aimed to determine the underlying causes and risk factors of chronic allograft dysfunction in renal transplant recipients.

Materials and Methods: From 2000 to 2012, all consecutive renal transplant recipients followed in our tertiary referral center who underwent renal biopsy due to an increase in serum creatinine level were enrolled. Etiologies of chronic allograft dysfunction were assessed according to pathologic results of renal biopsy specimens and laboratory findings. The immunological and non-immunological risk factors of chronic allograft dysfunction were screened and recorded retrospectively.

Results: Eighty (80) renal transplant recipients with a mean age of $38 \pm 10$ years were included in the study. Delayed graft function $(p=0.007)$, history of acute rejection $(p<0.001)$, positive panel reactive antibody $(p=0.033)$ (Class I $(p=0.013)$, Class II $(p=0.006))$, positive donor specific antibodies $(p=0.001)$, number of recurrent acute rejections $(p<0.001)$, number of human leukocyte antigens mismatches $(p=0.051)$, cold ischemia time $(p=0.001)$ were found to be risk factors for chronic allograft dysfunction. The donor specific antibodies positivity $(p<0.001)$ and the panel reactive antibody positivity (Class I $(p=0.003)$, Class II $(p=0.001))$ were significantly higher in patients with antibody mediated rejection than patients without antibody mediated rejection ( $p=0.002)$.

Conclusion: Delayed graft function, presence and the number of acute rejections, increased cold ischemia time, panel reactive antibody positivity, donor specific antibodies positivity, and the number of human leukocyte antigens mismatches were risk factors for chronic allograft dysfunction.

Keywords: Renal transplantation, chronic allograft dysfunction, risk factors, etiology. 


\section{INTRODUCTION}

Chronic kidney disease (CKD) is a serious health problem increasing both patient morbidity and mortality. It also induces a heavy burden on the national economy [1]. Based on the Turkish Society of Nephrology and the National Registry of Statistics Annual Report in 2019, the prevalence and the incidence of CKD were reported 1007 and 150.5 per million people, respectively. In Turkey, there have been 83783 patients with CKD, and this amount is increasing progressively [2]. Thus, prevention and management of CKD are of paramount importance [3].

The management of end-stage kidney disease is achieved with two distinct treatment modalities: dialysis and kidney transplantation [4]. Compared to the dialysis treatment, renal transplantation (RT) is better in improving life quality and patient outcomes if performed from a well-matched donor [5]. Therefore, RT is currently the most appropriate treatment option for end-stage kidney disease.

Over the last 30 years, the 1-year graft survival has been markedly increased depending on the advances in the immunosuppressive treatment modalities [6]. Notably, acute rejection was one of the most observed reasons for graft loss until the 1980s [7]. However, since the introduction of calcineurin inhibitors, acute rejection within 1-year after transplantation has decreased to less than $15 \%$, which led the 1-year graft survival to increase up to $90 \%$ and $95 \%$ for cadaveric and living transplantation, respectively $[8,9]$. On the other and, long-term graft survival has not reached the desired survival rates due to several immunological and non-immunological factors causing graft dysfunction and failure in the long term [10]. Consequently, recognition of the risk factors leading to long-term organ dysfunction has become the target of nephrologists.

Early detection of graft injury and identifying the underlying pathology can help to increase long-term graft survival. Kidney biopsy is the gold standard method for early detection of kidney damage; however, the invasiveness of the procedure limits its applicability [11]. In routine clinical practice, graft injury might clinically be recognized by the increase in serum creatinine and/ or development of proteinuria or hypertension, which are only detected after the injury has already occurred.
Considering that kidney graft function is monitored with serum creatinine and glomerular filtration rate in most centers, timely detection of graft injury is not always possible, and recognition of the injury could be delayed. Identification of causative factors would make it possible to recognize highrisk patients earlier, and to take preventive actions before the occurrence of graft damage. Therefore, the current study aimed to determine the risk factors and causes of chronic allograft dysfunction in patients undergoing renal transplantation.

\section{MATERIALS and METHODS}

This is a cross-sectional study conducted at the Nephrology Department of Hacettepe University Faculty of Medicine. All patients who underwent kidney biopsy following renal transplantation due to an increase in serum creatinine levels between 2000-2012 were included. Age under 18 years was the only exclusion criteria. Protocol transplant biopsy was not a routine procedure in our hospital and not included in the current study. Demographic, clinical, and laboratory data of all patients and donors were recorded before and after transplantation. The recorded parameters of the patients included the gender, age at the time of kidney transplantation, date of transplantation, etiology of end-stage kidney disease, comorbid diseases, time interval since transplantation to rejection, donor source (living vs. cadaveric), the number of transplantations, type and duration of pre-transplant dialysis, presence of preemptive transplantation, presence of delayed graft function, number and type of rejections, pre-transplant citomegalovirus IgM and IgG values, warm and cold ischemia times, renal biopsy results, pretransplant level of human leukocyte antigens (HLA) match, pretransplant panel reactive antibody (PRA) and donor specific antibodies (DSA) results. Also, recorded donor characteristics included the sex, age at the time of transplantation, cause of deaths of cadaveric donors, comorbid diseases, and level of HLA match before transplantation. In our center, we adopted PRA and DSA are positive if PRA $>5 \%$ and DSA $>1000 \mathrm{mfi}$, respectively. The data were obtained from the Hacettepe University Faculty of Medicine electronic database and the Nephrology Department's kidney transplant files. 
Factors causing graft dysfunction were grouped as immunological and non-immunological causes, and risk factors were determined. The current research was approved by Clinical Researches Ethics Boards of Hacettepe University (GO13-311) at 15 May 2013.

Statistical analyses were performed using SPSS version 20.0 (Chicago, USA). Variables were expressed as mean \pm standard deviation. Chi-square tests estimated differences in group rates. The risk factors that could cause graft dysfunction were tested by multivariate analysis and independent variables were determined. Groups were compared with the Mann Whitney $U$ test and chi-square tests. A p-value of $<0.05$ was considered statistically significant.

\section{RESULTS}

The demographic data of the patients was presented in Table-1. In total, 1080 kidney transplant patients were followed during the study period. Of all, eighty patients (52 male) were met the inclusion criteria and included in the study with a mean age of $38 \pm 10$ years. The mean follow-up period was $71 \pm 68$ months. Urological problems (vesicoureteral reflux, hydroureteronephrosis, neurogenic bladder, nephrolithiasis) were the most common reasons for primary renal dysfunction. The other leading etiologies were glomerulonephritis (Focal segmental glomerulosclerosis (FSGS), IgA nephropathy, membranoproliferative glomerulonephritis (MPGN), systemic lupus erythematosus (SLE) syndrome, polycystic kidney disease, and diabetic nephropathy). The etiology of end-stage kidney disease was unclear in 21 patients. Hypertension was the most comorbid disease $(49 \%)$, followed by coronary artery disease, diabetes mellitus, chronic obstructive pulmonary disease (COPD), SLE, malignancy (seminoma), cerebrovascular disease, and infectious diseases such as HBV, HCV, and tuberculosis (Table 1).

Cadaveric transplantation, previous history of kidney transplantation, preemptive transplantation, presence of pre-transplant dialysis, delayed graft dysfunction, history and number of rejections, prolonged hot and cold ischemia, diabetes mellitus, hypertension, CMV infection, vascular involvement, PRA and DSA positivity, the number of HLA mismatches were studied for being a risk factor. Presence of delayed graft function $(p=$ $0.007)$, history of acute rejection ( $p<0.001)$, number of recurrent acute rejections ( $p<0.001$ ), higher number of HLA mismatches $(p=0.05)$, increase in cold ischemia time $(p=0.001)$, positive PRA ( $p$ $=0.033)($ Class I $(P=0.013)$, Class II $(p=0.006))$, and positive DSA ( $p=0.001$ ) were found to be risk factors for chronic allograft dysfunction (Table 2).

The causes of graft dysfunction were assessed in two groups as immunological and non-immunological factors. Cellular and antibody mediated rejections were detected respectively in $23(26 \%)$ and 15 (19\%) patients among the patients with graft dysfunction due to immunological factors. On the other hand, non-immunological factors were found as follows; calcineurin inhibitor toxicity in 18 patients $(22.5 \%)$, recurrence of primary disease in 10 patients (12.5\%), hypertension-associated changes in 9 patients (11\%), tubulointerstitial nephritis in 7 patients (9\%), de novo glomerulonephritis in 6 patients $(7.5 \%)$ and BK virus nephropathy in 2 patients (2.5\%). Etiology of end-stage disease in rejected kidneys was undetermined in 4 patients $(5 \%)$, and 2 (2.5\%) patients could not be evaluated optimally due to insufficient renal biopsy specimens. Three patients had both cellular and antibody mediated rejections. The remaining 45 (56\%) patients had no signs of rejection (Figure 1 and Figure 2).

The duration of pre-transplant dialysis $(p<0.001)$, warm and cold ischemia time $(p=0.02$ and $p$ $<0.001)$, the number of recurrent rejections after transplantation $(p=0.007)$ were significantly higher in patients with cellular rejection than the patients with non-cellular rejection. Cellular rejection was more common in patients with cadaveric transplantation $(p=0.003)$, post-transplant rejection $(p<0.001)$, and delayed graft function $(p<0.001)$. The pre-transplant number of HLA mismatches was higher in patients with cellular rejection than in patients without cellular rejection; however, it did not reach statistically significance $(p=0.085)$. There was no statistical difference between the patients with and without cellular rejection in terms of PRA positivity $(p=0.64)$, hypertension $(p=0.85)$ or presence of diabetes $(p=1)$, prior transplantation $(p=0.33)$, preemptive transplantation $(p=0.23)$, post-transplant CMV infection $(p=0.18)$ and DSA positivity $(p=0.28)$ (Table 3$)$. 
Table 1. Demographic Data.

\begin{tabular}{|c|c|c|c|}
\hline & & Mean & n (\%) \\
\hline Age & & $38 \pm 10$ & - \\
\hline Duration after transplantation (month) & & $71 \pm 68$ & - \\
\hline Age of the donor during transplantation & & $45 \pm 15$ & - \\
\hline Duration of dialysis before transplantation (month) & & $34 \pm 53$ & - \\
\hline \multirow[t]{2}{*}{ Sex of the recipient } & Male & & $52(65)$ \\
\hline & Female & & $28(35)$ \\
\hline \multirow[t]{20}{*}{ Dialysis modality before transplantation } & Hemodialysis & & $66(82)$ \\
\hline & Peritoneal dialysis & & $6(7.5)$ \\
\hline & Peritoneal dialysis + Hemodialysis & & $8(10.5)$ \\
\hline & Unknown & & $21(26.3)$ \\
\hline & Urogenital disease & & $20(25)$ \\
\hline & Glomerulonephritis & & $20(25)$ \\
\hline & -FSGS & & $7(8.8)$ \\
\hline & -IgA nephropathy & & $3(3.8)$ \\
\hline & -MPGN & & $3(3.8)$ \\
\hline & $-S L E$ & & $2(2.5)$ \\
\hline & -Post-infection GN & & $2(2.5)$ \\
\hline & -Goodpasture syndrome & & $1(1.3)$ \\
\hline & -Membraneous glomerulonephritis & & $1(1.3)$ \\
\hline & $-\mathrm{HSP}$ & & $1(1.3)$ \\
\hline & Hypertension & & $8(10)$ \\
\hline & Amyloidosis & & $5(6.3)$ \\
\hline & Congenital disease & & $5(6.3)$ \\
\hline & -Alport syndrome & & $3(3.8)$ \\
\hline & -Polycystic renal disease & & $2(2.5)$ \\
\hline & Diabetic nephropathy & & $1(1.3)$ \\
\hline \multirow[t]{11}{*}{ Comorbid disease } & Hypertension & & $39(48.8)$ \\
\hline & Chronic Infection & & $11(13.8)$ \\
\hline & $-\mathrm{HCV}$ & & $6(7.5)$ \\
\hline & $-H B V$ & & $3(3.8)$ \\
\hline & -Tuberculosis & & $2(2.5)$ \\
\hline & Coronary Artery Disease & & $5(6.3)$ \\
\hline & CVA & & $1(1.3)$ \\
\hline & Diabetes & & $2(2.5)$ \\
\hline & COPD & & $2(2.5)$ \\
\hline & SLE & & $1(1.3)$ \\
\hline & Malignancy & & $1(1.3)$ \\
\hline
\end{tabular}

FSGS: Focal segmental glomerulosclerosis, IgA: Immunoglobulin A, MPGN: Membranoproliferative glomerulonephritis, SLE: Systemic Lupus Erythematosus, GN: glomerulonephritis, HSP: Henoch-Schönlein purpura, HCV: hepatitis C virus infection, HBV: hepatitis B infection, CVA: cerebrovascular accident, COPD: Chronic Obstructive Pulmonary Disease

The DSA positivity $(p<0.001)$ and the PRA positivity (Class I $(P=0.003)$, Class II $(p=0.001))$ were significantly higher in patients with antibody mediated rejection than patients without antibody mediated rejection $(p=0.02)$. Besides, presence of earlier rejection $(p=0.007)$ and number of recurrent rejections $(p=0.001)$ were significantly higher in patients with antibody mediated rejection than patients without any rejection. However, these significant differences in the univariate analyzes has lost significance in multivariate analyzes. The post-transplant incidence of CMV infection was not statistically different between patients with antibody mediated and cellular rejection, despite 
Table 2. Comparison of the risk factors of chronic allograft dysfunction due to immunologic and non-immunologic reasons.

\begin{tabular}{|c|c|c|c|}
\hline & Immunologic n (\%) & Non-immunologic $n(\%)$ & $\mathrm{p}$ \\
\hline Cadaveric transplantation & $13(37)$ & $10(22)$ & 0.14 \\
\hline Having previous transplantation & $3(9)$ & $1(2)$ & 0.21 \\
\hline Preemptive transplantation & $3(9)$ & $5(12)$ & 0.608 \\
\hline Dialysis before transplantation & $32(91)$ & $40(87)$ & 0.55 \\
\hline Delayed graft dysfunction & $15(43)$ & $5(12)$ & 0.007 \\
\hline Rejection & $30(86)$ & $9(20)$ & $<0.001$ \\
\hline Rejection number & $1(1 ; 3)$ & $0(0 ; 1)$ & $<0.001$ \\
\hline Cold Ischemia Time & $630(50 ; 1080)$ & $157(37 ; 277)$ & 0.001 \\
\hline Warm Ischemia Time & $3.04(2 ; 4)$ & $2.33(2 ; 3)$ & 0.14 \\
\hline Diabetes & $3(9)$ & $4(10)$ & 0.95 \\
\hline Hypertension & $20(57)$ & $30(67)$ & 0.38 \\
\hline CMV infection & $2(6)$ & $2(5)$ & 0.83 \\
\hline Vascular involvement & $19(54)$ & $3(7)$ & $<0.001$ \\
\hline PRA I positivity & $13(37)$ & $6(13)$ & 0.013 \\
\hline PRA II positivity & $19(54)$ & $11(24)$ & 0.006 \\
\hline PRA positivity & $20(57)$ & $15(33)$ & 0.033 \\
\hline DSA positivity & $14(40)$ & $4(9)$ & 0.001 \\
\hline HLA missmatch number & $2(2 ; 3)$ & $2(1 ; 3)$ & 0.05 \\
\hline
\end{tabular}

CMV: Cytomegalovirus, PRA: Panel Reactive Antibody, DSA: Donor Specific Antibody, HLA: Human Leukocyte Antigen

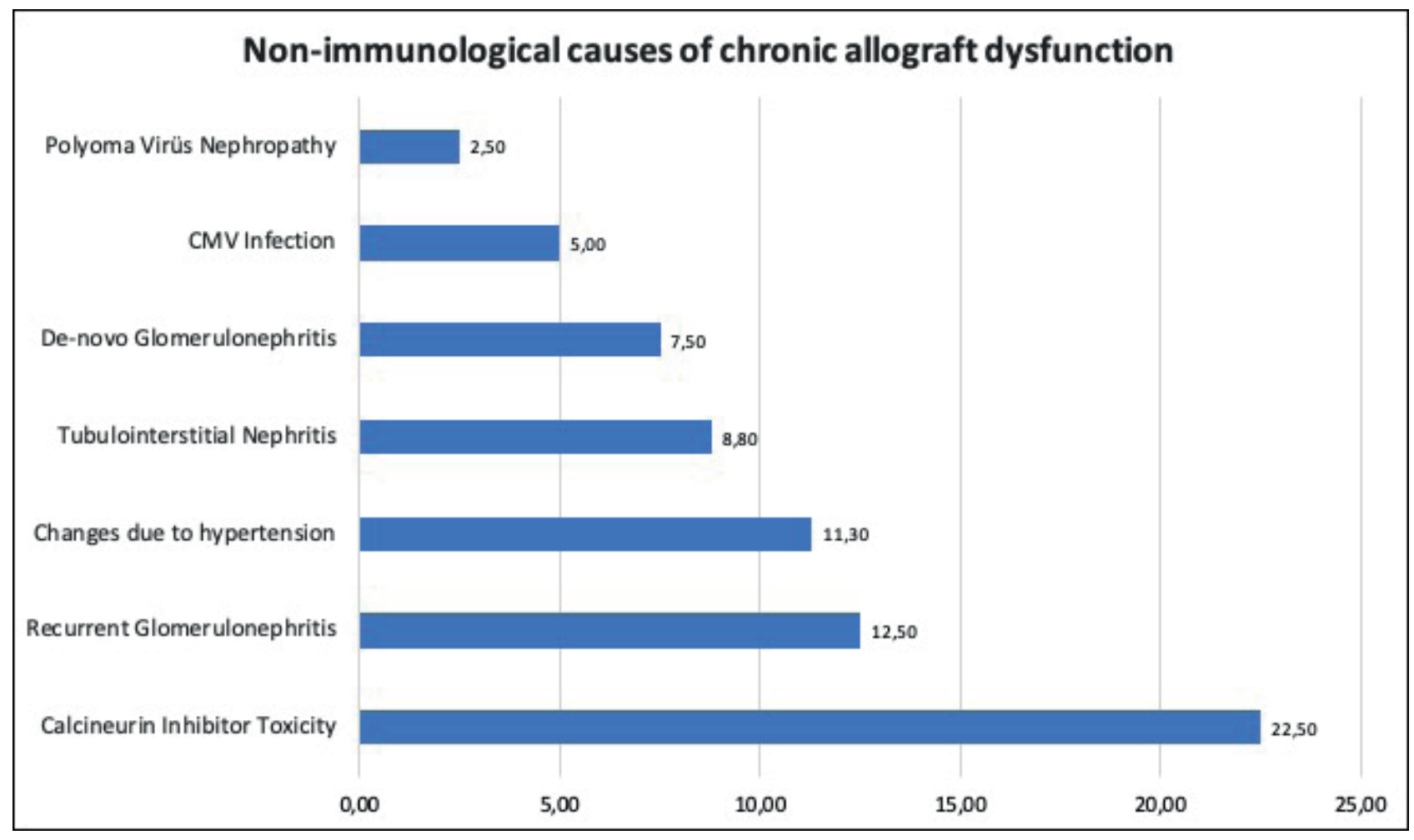

Figure 1. Non-immunological causes of chronic allograft dysfunction.

tending to a significant level $(p=0.083)$. Patients with and without antibody mediated rejection were statistically similar in terms of having hypertension $(p=0.82)$ and diabetes $(p=0.75)$, transplants from living or cadaveric donors $(p=0.44)$, previous history of transplantation ( $p=0.13)$, delayed graft function $(p=0.24)$ and preemptive transplantation $(p=0.64)$ (Table 3$)$. 


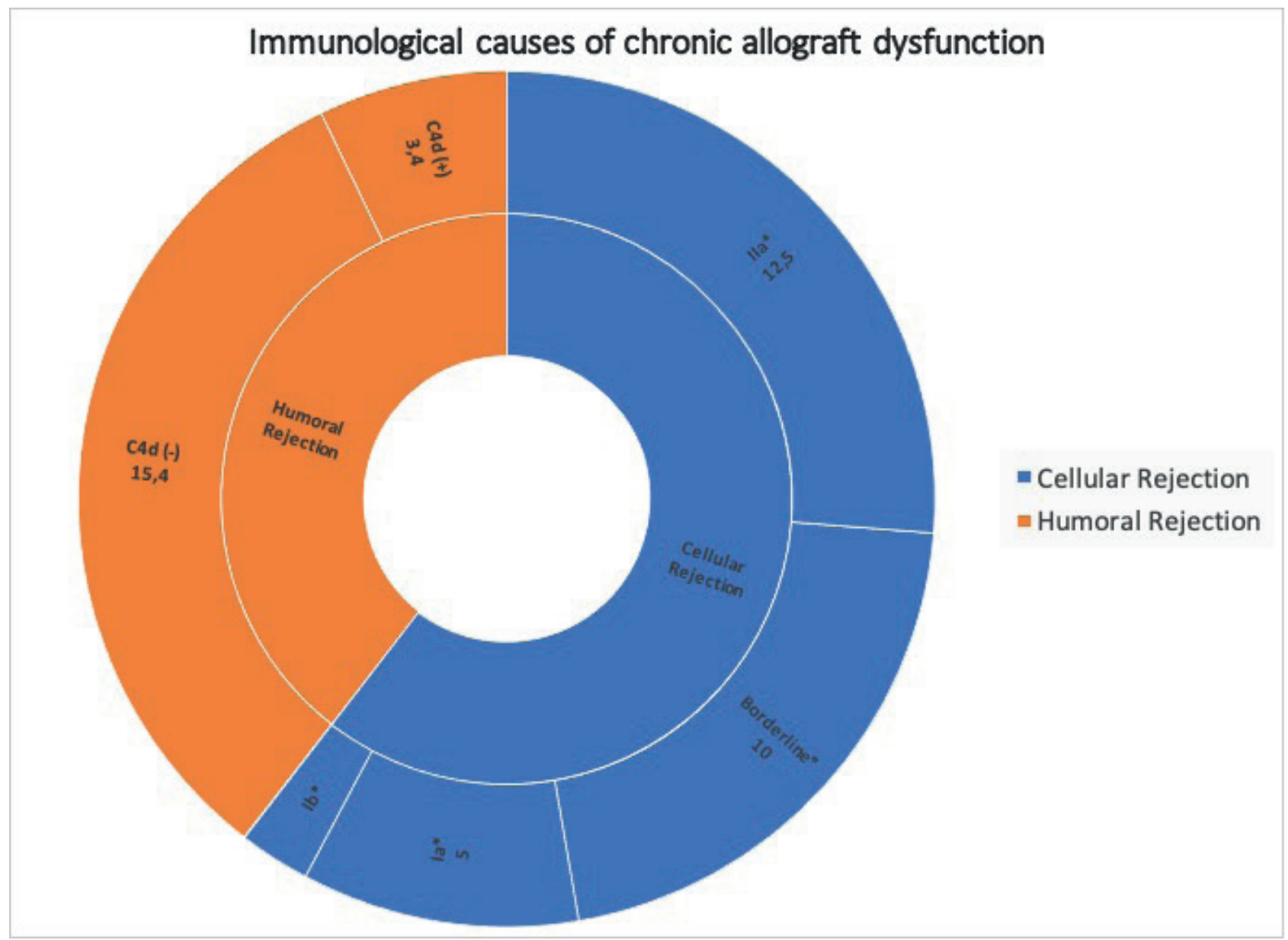

Figure 2. Immunological causes of chronic allograft dysfunction.

(*) Banff 97 diagnostic criterias for renal allograft biopsies (updated in 2007)

Table 3. Comparison of risk factors in patients with cellular and antibody mediated rejection.

\begin{tabular}{|l|c|c|c|c|c|c|}
\hline \multirow{2}{*}{} & \multicolumn{3}{|c|}{ Cellular Rejection } & \multicolumn{3}{c|}{ Antibody mediated Rejection } \\
\cline { 2 - 7 } & Positive (\%) & Negative (\%) & $p$ & Positive (\%) & Negative (\%) & $p$ \\
\hline Cadaveric transplantation & $4(17)$ & $19(83)$ & 0.003 & $4(21)$ & $14(79)$ & 0.44 \\
History of previous transplantation & $11(48)$ & $12(52)$ & 0.33 & $9(50)$ & $9(50)$ & 0.13 \\
Preemptive transplantation & $3(12,5)$ & $20(87,5)$ & 0.23 & 25 & 75 & 0.64 \\
Dialysis before transplantation & $8(33)$ & $15(67)$ & 0.23 & $4(21)$ & $14(79)$ & 0.64 \\
Delayed graft dysfunction & $17(75)$ & $6(25)$ & $<0.001$ & $1(6)$ & $17(94)$ & 0.24 \\
Rejection & $12(52)$ & $11(48)$ & $<0.001$ & $6(33)$ & $12(67)$ & 0.007 \\
Rejection number & $1(1 ; 1)$ & $0(0 ; 1)$ & 0.007 & $1(0.25 ; 1)$ & $0(0 ; 1)$ & 0.001 \\
Cold Ischemia Time & $855(50 ; 1125)$ & $35(30 ; 60)$ & $<0.001$ & $57.5(41 ; 1050)$ & $50(30 ; 784)$ & 0.81 \\
Warm Ischemia Time & $3(2 ; 5)$ & $2.5(2 ; 3)$ & 0.021 & $2.5(1.8 ; 2.9)$ & $2.7(2 ; 3)$ & 0.55 \\
Diabetes & $7(30)$ & $16(70)$ & 1 & $3(17)$ & $15(83)$ & 0.752 \\
Hypertension & $7(30)$ & $16(70)$ & 0.85 & $4(21)$ & $14(79)$ & 0.821 \\
CMV infection & 0 & $23(100)$ & 0.18 & $9(50)$ & $9(50)$ & 0.083 \\
Vascular involvement & $16(68)$ & $7(32)$ & $<0.001$ & $6(33)$ & $12(67)$ & 0.11 \\
PRA I positivity & $9(37)$ & $14(63)$ & 0.37 & $8(44)$ & $10(56)$ & 0.003 \\
PRA II positivity & $9(37)$ & $14(63)$ & 0.23 & $7(38)$ & $11(62)$ & $\mathbf{0 . 0 0 1}$ \\
PRA positivity & $7(30)$ & $16(70)$ & 0.64 & $6(33)$ & $12(67)$ & 0.002 \\
DSA positivity & $7(30)$ & $16(70)$ & 0.28 & $12(67)$ & $6(33)$ & $<0.001$ \\
HLA missmatch number & $2(1 ; 3)$ & $2(1 ; 3)$ & 0.085 & $2(0.5 ; 3.5)$ & $2(1 ; 3)$ & 0.642 \\
\hline
\end{tabular}

CMV: Cytomegalovirus, PRA: Panel Reactive Antibody, DSA: Donor Specific Antibody, HLA: Human Leukocyte Antigen 
PRA was positive in 18 patients (22.5\%), 31 patients (39\%), and 14 patients (17.5\%) for Class I antigens, Class II antigens and both Class I and Class II antigens, respectively. In total, PRA positivity was found in 35 (44\%) patients. DSA was positive in 18 (22.5\%) patients with PRA positivity, while DSA was found to be positive in $51 \%$ of the PRA positive group. C4d positivity was detected in $80 \%(n=12)$ of patients with antibody mediated rejection. In two patients, C4d positivity could not be shown even though findings were supporting antibody mediated rejection. PRA was positive in $80 \%$ of patients with antibody mediated rejection. Besides, $83 \%$ of the patients with antibody mediated rejection and PRA positivity were also DSA positive. DSA positive patients represented $67 \%$ of the patients having antibody mediated rejection. However, DSA positivity was found $17 \%$ of patients with cellular rejection, and $9 \%$ of patients with chronic allograft dysfunction due to non-immunological reasons.

Statistically, DSA positivity was higher in patients with antibody mediated rejection than other causes $(p<0.001)$. However, there was no similar relationship in patients with cellular rejection ( $p$ $=0.28$ ). DSA positivity was significantly lower in patients with non-immunological caused rejections $(p=0.001)$. A multivariable model was applied to the parameters that were found statistically significant in univariate analysis. However, none of these parameters were found significant considering whole cohort.

\section{DISCUSSION}

In the current study, delayed graft function, history and the number of acute rejections, duration of cold ischemia, presence of vascular involvement, PRA positivity (Class I and Class II), DSA positivity, increased number of HLA mismatches were determined as risk factors for the development of chronic allograft dysfunction. Cellular and antibody mediated rejections, calcineurin inhibitor toxicity, recurrent and de novo glomerulonephritis, tubulointerstitial nephritis, hypertension, BK nephropathy were all found as the causes of chronic allograft dysfunction.

In parallel with the previous studies, delayed graft function and the increase of cold ischemia time were found to be risk factors for chronic allograft dysfunction. The increase in cold ischemia time and delayed graft function was significantly higher in patients with cellular rejection than the patients with non-cellular rejection. A similar relationship could not be demonstrated in patients with chronic allograft dysfunction secondary to antibody mediated rejection. The reason behind that could be the activation of T-cell immunity towards the mediators, which are secreted after ischemia and tissue damage [12]. In the literature, it has already been shown that delayed kidney function negatively affects the graft survival in both early and chronic terms. Quiroga et al. reported that the development of delayed graft function and increase of cold ischemia time impair the graft survival in early and chronic periods in 518 RT recipients [3]. Similarly, in a study done by Woo et al. including 32,557 RT recipients, the delayed graft function and increase in cold ischemia time were found to affect graft survival adversely, especially if the donors were older than 55 years [5].

The occurrence and number of acute rejections have also been identified as risk factors for developing chronic allograft dysfunction. Almond and colleagues investigated the risk factors for developing chronic rejection in 566 patients with RT. They examined the impact of previous kidney transplantation, age, gender, HLA mismatch count, PRA positivity, acute rejection, infection, donor age, and cyclosporine dosage on chronic rejection in their five-year follow-up study. As a result, they reported that acute rejection, infection, and cyclosporine dosage below $5 \mathrm{mg} / \mathrm{kg} /$ day were major risk factors for developing chronic rejection [13]. Similarly, Meier et al. also showed that acute rejection is an independent risk factor for chronic allograft dysfunction in 63045 patients [8]. In parallel to the studies of Meier and Arnold, McDonald et al. showed that acute rejection within three months following kidney transplantation has a significant impact on chronic allograft dysfunction in a study including $4325 \mathrm{RT}$ recipients [9].

Unsurprisingly, the antibody mediated rejection was significantly higher in patients with antiHLA antibodies than patients without anti-HLA antibodies. Anti-HLA antibody positivity was found significantly lower in chronic allograft dysfunction with non-immunological reasons. Additional studies also show that anti-HLA antibodies were associated with chronic allograft dysfunction. Mao 
and colleagues conducted a 5-year follow-up study on 54 kidney transplant patients. They reported three graft rejections among 22 anti-HLA antibodynegative patients as opposed to 17 patients among 32 anti-HLA antibody-positive patients. If the creatinine cut-off level was assigned higher than $4 \mathrm{mg} / \mathrm{dl}$ to define graft loss, 13 of 15 DSA positive patients developed graft loss compared to 4 out of 22 patients with a negative anti-HLA antibody [14]. The main point of this study was that anti-HLA antibody positivity was detected before an increase in serum creatinine in patients progressing to graft loss. In another study performed with 120 nonsensitized RT recipients, Piazza et al. showed that acute rejection and graft loss were more common in DSA positive patients [13].

Anti HLA antibodies can already exist or appear after transplantation. The presence of AntiHLA antibodies before kidney transplantation negatively affects graft survival. In parallel, antiHLA antibodies emerging after transplantation negatively affect graft survival. In a study by Lachmann et al., anti-HLA antibody positivity was 30\% within 1014 patients with cadaveric transplants. One-third of these patients had DSA positivity, and graft survival was significantly lower in this group. A prospective study, including 195 $\mathrm{RT}$ recipients, the graft survival decreased as the anti-HLA antibody became positive despite being negative before transplantation [15]. Overall, under the light of these studies, the development of antiHLA antibody negatively affects graft survival. Unfortunately, there is no clear data regarding when and how often to check anti HLA antibodies in these patients. Surprisingly, some cases can have normal graft function despite having positive anti-HLA antibody. Lee et al. checked for anti-HLA antibodies in $139 \mathrm{RT}$ recipients at the 3rd, 6th, 12th months, and annually thereafter transplantation. Twenty-nine patients developed chronic rejection, and anti-HLA antibody positivity was detected before rejection in all of these patients. On the other hand, $27 \%$ of 110 patients developed anti-HLA antibodies despite having normal graft function [16]. In multivariate analysis, the development of anti-HLA antibody alone was not a risk factor for rejection, which is attributed to having a small number of study population. The presence of PRA positivity in patients with graft dysfunction due to immunological reasons may indicate that monitoring anti HLA antibody may be beneficial inpatient follow-up.

Cadaver transplantation, presence of previous transplantation, preemptive transplantation, dialysis before transplantation, diabetes, hypertension, and CMV infections were not revealed as risk factors in the current study, which may be due to insufficient number of patients. Cadaver transplantation, a history of previous transplantation, and dialysis before kidney transplantation were reported as risk factors for developing chronic allograft dysfunction in large populations [17]. In case of enrolling a sufficient number of patients, we assume consistent results with these studies.

The current study showed that calcineurin inhibitor (CNI) toxicity (22.5\%) is the leading non-immunological cause of chronic allograft dysfunction. In accordance with the current study, CNI toxicity has been found as the most common non-immunological cause of chronic allograft dysfunction in other studies [18-20]. In a prospective study performed on 961 patients, Nankivell et al. reported that cyclosporine toxicity was the reason in $15 \%, 65 \%$, and $100 \%$ of graft rejections at the first, fifth, and 10th years following kidney transplantation, respectively. The dosage is an important factor in the development of cyclosporine toxicity [21]. The risk of acute rejection increases if the CNI dose is insufficient. Conversely, excess $\mathrm{CNI}$ dose causes toxicity and graft injury. Therefore, the use of CNIs is like a two-edged sword, and chronic allograft dysfunction might develop in both lower and higher than required doses.

Relapsing glomerulonephritis was the $2 \mathrm{nd}$, and de novo glomerulonephritis was the 5 th most frequent non-immunological causes of graft injury in our study, which developed in 10 (12.5\%) and 6 (7.5\%) patients, respectively. These rates are compatible with previous studies. In a study performed by Briganti et al. between 1988-1997, patients with kidney transplantation were followed for ten years. It was revealed that relapsing glomerulonephritis was the reason behind $8.4 \%$ of graft losses [22]. In a study aimed to demonstrate the causes of kidney allograft loss, El-Zoghby et al. showed that $14.3 \%$ of patients had graft dysfunction due to relapsing glomerulonephritis and $6.6 \%$ due to de novo glomerulonephritis, among 1317 RT recipients. 
Hypertension was the third cause among the nonimmunological reasons of graft dysfunction in our cohort. However, the presence of hypertension was not shown as a risk factor for chronic allograft dysfunction. In parallel with our study, Kasiske and colleagues reported that graft function was negatively affected by increased blood pressure; however, hypertension was not an independent risk factor for chronic allograft dysfunction [23].

In the current study, four patients were infected with CMV, and two patients with BK virus. While CMV infection did not cause graft dysfunction, BK nephropathy caused graft dysfunction in 2 patients. Polyomavirus nephropathy (BK virus) may cause graft dysfunction and loss, which should be considered in the differential diagnosis of graft dysfunction $[24,25]$.

The generalizability of our findings is subject to certain limitations. A major limitation is the relatively small number of patients included. Secondly, this study has a retrospective design, which might be associated with missing data. Thirdly, these findings represent the results of a single center, although our center is one of the biggest referral centers in the country. Fourthly, drug compliance has not been formally checked in our department and we were not able to assess the distinction between the drug compliant and non-compliant.

\section{CONCLUSION}

In conclusion, this study has revealed that delayed graft function, presence and the number of previous acute rejections, increased cold ischemia time, PRA positivity, DSA positivity, and the number of HLA mismatches were risk factors for having chronic renal allograft dysfunction. Monitoring of the defined immunological factors in regular intervals might help to predict chronic allograft nephropathy earlier. Further research in this field would be of great help in order to understand if monitoring these parameters would have an influence on long-term graft survival.

\section{CONFLICT Of INTEREST STATEMENT}

The authors declare that there is no conflict of interest.

\section{reO REFERENCES Ceen}

[1] Bikbov B, Purcell CA, Levey AS, et al. Global, regional, and national burden of chronic kidney disease, 1990-2017: a systematic analysis for the Global Burden of Disease Study 2017. The Lancet. 2020; 395(10225): 709-33.

[2] Seyahi N, Ates K, Suleymanlar G. Current Status of Renal Replacement Therapy in Turkey: A Summary of the Turkish Society of Nephrology Registry Report. Turk J Nephrol. 2020; 29(1): 6-11.

[3] Quiroga I, McShane P, Koo DDH, et al. Major effects of delayed graft function and cold ischaemia time on renal allograft survival. Nephrol Dial Transplant. 2006; 21(6): 1689-96.

[4] Queeley GL, Campbell ES. Comparing treatment modalities for end-stage renal disease: a meta-analysis. Value Health. 2014; 17(3): A290.

[5] Woo YMUN, Gill JS, Johnson N, et al. The advanced age deceased kidney donor: Current outcomes and future opportunities. Kidney Int. 2005; 67(6): 2407-14.

[6] Shrestha B, Haylor J, Raftery A. Historical Perspectives in Kidney Transplantation: An Updated Review. Prog Transplant. 2015; 25(1): 64-76. Hariharan S, Mcintosh MJ. Improved graft survival after renal transplantation in the United States, 1988 to 1996. N Engl J Med. 2000; 8.
[7] Meier-Kriesche H-U, Ojo AO, Hanson JA, et al. Increased impact of acute rejection of acute rejection on chronic allograft failure in recent era: Transplantation. 2000; 70(7): 1098-100.

[8] McDonald S, Russ G, Campbell S, et al. Kidney Transplant Rejection in Australia and New Zealand: Relationships Between Rejection and Graft Outcome. Am J Transplant. 2007; 7(5): 1201-8.

[9] Pascual M, Theruvath T, Kawai T, et al. Strategies to Improve Long-Term Outcomes after Renal Transplantation. N Engl J Med. 2002; 346(8): 580-90.

[10] Ahmad I. Biopsy of the Transplanted Kidney. Semin Interv Radiol. 2005; 21(04): 275-81.

[11] Ponticelli CE. The impact of cold ischemia time on renal transplant outcome. Kidney Int. 2015; 87(2): 272-5.

[12] Piazza A, Poggi E, Borrelli L, et al. Impact of donor-specific antibodies on chronic rejection occurrence and graft loss in renal transplantation: Posttransplant analysis using flow cytometric techniques. Transplantation. 2001;71(8): 1106-12.

[13] Mao Q, Terasaki PI, Cai J, et al. Extremely High Association Between Appearance of HLA Antibodies and Failure of Kidney Grafts in a Five-Year Longitudinal Study. Am J Transplant. 2007; 7(4): 864-71. 
[14] Lachmann N, Terasaki PI, Budde K, et al. Anti-Human Leukocyte Antigen and Donor-Specific Antibodies Detected by Luminex Posttransplant Serve as Biomarkers for Chronic Rejection of Renal Allografts: Transplantation. 2009; 87(10): 1505-13.

[15] Lee P-C, Terasaki PI, Takemoto SK, et al. All chronic rejection failures of kidney transplants were preceded by the development of HLA antibodies: Transplantation. 2002; 74(8): 1192-4.

[16] Knoll G. Canadian Society of Transplantation: consensus guidelines on eligibility for kidney transplantation. Can Med Assoc J. 2005; 173(10): S1-25.

[17] Mathis AS, Egloff G, Ghin HL. Calcineurin inhibitor sparing strategies in renal transplantation, part one: Late sparing strategies. World J Transplant. 2014; 4(2): 57.

[18] Panek R, Tennankore KK, Kiberd BA. Incidence, etiology, and significance of acute kidney injury in the early postkidney transplant period. Clin Transplant. 2016; 30(1): 66-70.
[19] Sawinski D, Trofe-Clark J, Leas B, et al. Calcineurin Inhibitor Minimization, Conversion, Withdrawal, and Avoidance Strategies in Renal Transplantation: A Systematic Review and Meta-Analysis. Am J Transplant. 2016; 16(7): 2117-38.

[20] Nankivell BJ, Borrows RJ, Fung CL-S, et al. Natural History, Risk Factors, and Impact of Subclinical Rejection in Kidney Transplantation. Transplant J. 2004; 78(2): 242-9.

[21] Briganti EM, Russ GR, McNeil JJ, et al. Risk of Renal Allograft Loss from Recurrent Glomerulonephritis. N Engl J Med. 2002; 347(2): 103-9.

[22] Kasiske BL, Anjum S, Shah R, et al. Hypertension after kidney transplantation. Am J Kidney Dis. 2004; 43(6): 1071-81.

[23] Barraclough KA, Isbel NM, Staatz CE, Johnson DW. BK Virus in Kidney Transplant Recipients: The Influence of Immunosuppression. J Transplant. 2011; 1-9.

[24] Lee S, Lee KW, Kim SJ, et al. Clinical Characteristic and Outcomes of BK Virus Infection in Kidney Transplant Recipients Managed Using a Systematic Surveillance and Treatment Strategy. Transplant Proc. 2020; 52(6): 1749-56. 